\title{
A systematic review of metabolite profiling in gestational diabetes mellitus
}

\author{
Jennifer Huynh • Grace Xiong • Rhonda Bentley-Lewis
}

Received: 27 May 2014 / Accepted: 14 August 2014 / Published online: 6 September 2014

(C) Springer-Verlag Berlin Heidelberg 2014

\begin{abstract}
Aims/hypothesis Gestational diabetes mellitus is associated with adverse maternal and fetal outcomes during, as well as subsequent to, pregnancy, including increased risk of type 2 diabetes and cardiovascular disease. Because of the importance of early risk stratification in preventing these complications, improved first-trimester biomarker determination for diagnosing gestational diabetes would enhance our ability to optimise both maternal and fetal health. Metabolomic profiling, the systematic study of small molecule products of biochemical pathways, has shown promise in the identification of key metabolites associated with the pathogenesis of several metabolic diseases, including gestational diabetes. This article provides a systematic review of the current state of research on biomarkers and gestational diabetes and discusses the clinical relevance of metabolomics in the prediction, diagnosis and management of gestational diabetes.

Methods We conducted a systematic search of MEDLINE (PubMed) up to the end of February 2014 using the key term combinations of 'metabolomics,' 'metabonomics,' 'nuclear magnetic spectroscopy,' 'mass spectrometry,' 'metabolic profiling' and 'amino acid profile' combined (AND) with 'gestational diabetes'. Additional articles were identified through searching the reference lists from included studies. Quality assessment of included articles was conducted through the use of QUADOMICS.

Results This systematic review included 17 articles. The biomarkers most consistently associated with gestational diabetes were asymmetric dimethylarginine and NEFAs. After
\end{abstract}

Electronic supplementary material The online version of this article (doi:10.1007/s00125-014-3371-0) contains peer-reviewed but unedited supplementary material, which is available to authorised users.

J. Huynh $\cdot$ G. Xiong $\cdot$ R. Bentley-Lewis $(\bowtie)$

Department of Medicine/Diabetes Unit, Massachusetts General

Hospital, 55 Fruit Street, Bulfinch 4-415, Boston, MA 02114, USA

e-mail: rbentleylewis@mgh.harvard.edu
QUADOMICS analysis, 13 of the 17 included studies were classified as 'high quality'.

Conclusions/interpretation Existing metabolomic studies of gestational diabetes present inconsistent findings regarding metabolite profile characteristics. Further studies are needed in larger, more racially/ethnically diverse populations.

Keywords Amino acids - Gestational diabetes mellitus . Metabolite profiling - Metabolites - Metabolomics .

Systematic review

$\begin{array}{ll}\text { Abbreviations } \\ \text { ADMA } & \text { Asymmetric dimethylarginine } \\ \text { BCAA } & \text { Branched-chain amino acid } \\ \text { CVD } & \text { Cardiovascular disease } \\ \text { GDM } & \text { Gestational diabetes mellitus } \\ \text { IADPSG } & \text { International Association of Diabetes and } \\ & \begin{array}{l}\text { Pregnancy Study Groups } \\ \text { LC-MS }\end{array} \\ \text { Liquid chromatography-mass spectrometry } \\ \text { NGT } & \text { Normal glucose tolerance } \\ \text { NMR } & \text { Nuclear magnetic resonance }\end{array}$

Introduction

Gestational diabetes mellitus (GDM), defined as diabetes diagnosed during pregnancy that is not clearly overt diabetes, affects from $5-6 \%$ to $15-20 \%$ of pregnancies in the USA, depending on population demographics, screening methodology and diagnostic criteria in use [1]. Several risk factors have been found to correlate highly with GDM, including advanced maternal age, race/ethnicity, obesity and family history of type 2 diabetes [2]. Studies have also shown that a pregnancy complicated by GDM is a significant risk factor for the subsequent development of type 2 diabetes [1] and cardiovascular disease (CVD) [3-5]. Although healthcare providers agree on 
the importance of diagnosing GDM, current risk assessment protocols recommended by professional societies vary. Consequently, healthcare providers are inclined to follow the recommendations from the professional society with which they are most closely aligned. Guidelines from the International Association of Diabetes and Pregnancy Study Groups (IADPSG) recommend that a one-step $2 \mathrm{~h}, 75 \mathrm{~g}$ OGTT be performed between 24 and 28 weeks' gestational age [1]. In contrast, the National Institutes of Health (NIH) and the American College of Obstetrics and Gynecology (ACOG) suggest a two-step diagnostic approach. Women who meet or exceed the screening threshold after a $1 \mathrm{~h}, 50 \mathrm{~g}$ oral glucose load test next undergo a $3 \mathrm{~h}, 100 \mathrm{~g}$ OGTT between 24 and 28 weeks' gestational age [1]. The ADA, which previously endorsed the one-step approach, now considers both methods acceptable for GDM screening [1]. The ADA encourages further research in this area because evidence definitively demonstrating the superiority of either approach is lacking.

Given the escalating national burden of type 2 diabetes [1] and CVD [6], earlier identification of GDM is critical to providing an opportunity for the application of primary prevention strategies. However, prior efforts to identify firsttrimester biomarkers and risk algorithms for subsequent GDM diagnosis have been limited. Associations have been found between levels of the following first-trimester biomarkers and increased risk of GDM: low follistatin-3 [7]; low sex hormone-binding globulin $[8,9]$; high C-reactive protein [9, 10]; and high tissue plasminogen activator and low HDL-cholesterol [11]. Although these models had variable degrees of predictive power based on the choice of clinical variables or biochemical surrogates of adiposity, none explored first-trimester metabolites to identify women at risk of GDM.

Metabolomics, the science of systematically constructing metabolite profiles to study metabolic pathways, has shown promise in the identification of novel pathways and early biomarkers indicative of insulin resistance and type 2 diabetes $[12,13]$. Metabolomic detection, identification and quantification are typically carried out by liquid chromatographymass spectrometry (LC-MS), gas chromatography-mass spectrometry (GC-MS) or nuclear magnetic resonance (NMR) spectroscopy [12], all highly sensitive metabolomic techniques. MS, which uses an analyser within an electromagnetic field, first allows the separation of ions within an analyte according to their mass-to-charge ratio. The detection of specific metabolite classes is possible when MS is coupled with gas or liquid chromatography, allowing analytical compound separation. Conversely, NMR spectroscopy uses a strong magnetic field to determine the structural and chemical properties of relevant molecules, allowing the identification of single metabolites and quantification of metabolite levels [12].

The current literature demonstrates the use of metabolomic profiling in the assessment of plasma, serum, urine, breast milk or amniotic fluid in the fasting, post-glucose load or postprandial state [14]. Recent investigations have also suggested combining metabolomics and the OGTT as an effective method of revealing unique metabolic phenotypes [15-17]. Metabolomic analyses of samples from participants before and after an OGTT have been used to detect early shifts in metabolism during the progression from early insulin resistance to type 2 diabetes [15]. Consequently, metabolomics can potentially improve on current diagnostic methods for GDM that focus solely on data from the OGTT.

Detailed reviews of metabolomic studies in type 2 diabetes have been presented elsewhere $[12,13]$ and have implicated specific clusters of metabolites, particularly among branchedchain and aromatic amino acids in the pathogenesis of type 2 diabetes. These findings point to perturbations in normal metabolism and allow the potential identification of novel pathways in the development of diabetes, thereby enriching our understanding of metabolic syndromes. The purpose of our review is to critically examine the clinical studies that both characterise metabolite profiles to predict GDM and distinguish metabolite profiles of women with GDM from those without GDM. Patterns of metabolic pathways found in the literature, as well as directions for future research, will be discussed in the context of metabolomics as a potential diagnostic tool for GDM.

\section{Methods}

Search strategy

Literature searches of MEDLINE (PubMed) for relevant articles were conducted up to the end of February 2014 with the key term combinations of 'metabolomics', 'metabonomics', 'nuclear magnetic spectroscopy', 'mass spectrometry', 'metabolic profiling' and 'amino acid profile' combined with 'gestational diabetes'. To minimise selection bias, two investigators (J. Huynh and G. Xiong) independently reviewed titles, abstracts and available full-text articles for relevance. Additional articles were identified through searching the reference lists from included studies. Disagreements were resolved by consensus and by a third investigator (R. Bentley-Lewis).

\section{Eligibility criteria}

Articles were included or excluded on the basis of full-text articles. The following prespecified inclusion criteria were applied: (1) participants included pregnant women classified as having GDM; (2) the control population was specified (e.g. women with normal glucose tolerance [NGT]); (3) metabolomic techniques such as LC-MS or NMR spectroscopy were used to construct metabolite profiles; and (4) metabolites were examined in maternal plasma, serum, urine or 
amniotic fluid. Studies were excluded if they (1) analysed metabolite profiles in animals or (2) did not have an appropriate control group.

\section{Data extraction and analysis}

Data on population characteristics and metabolomic techniques and study results were extracted. One investigator performed the data extraction (J. Huynh), which was verified by a second investigator (R. bentley-Lewis). Owing to the limited number of studies relevant to GDM and metabolomics, the substantial methodological heterogeneity and the considerable variations in study population characteristics, a quantitative meta-analysis of the data was not appropriate.

Methodological quality assessment

The study team used QUADOMICS to assess the methodological quality of the studies. QUADOMICS - an adaptation of QUADAS, a quality assessment tool used in systematic reviews of diagnostic accuracy studies - was developed to assess quality issues specific to '-omics' research, including the quality assessment of studies included in systematic reviews [18]. The methodologies of studies that achieved 12/16 or more on the QUADOMICS tool were classified as 'high quality', whereas those that scored 11/16 or lower were classified as 'low quality'.

\section{Results}

\section{Study characteristics}

The selection algorithm for the 17 studies that met the inclusion/exclusion criteria for our systematic review is detailed in Fig. 1. All included studies compared metabolites in women with GDM with those in women with NGT. The sample size of the GDM study population varied among the studies, ranging from eight to 79 participants. In addition, the GDM diagnostic criteria used varied among the studies. Two studies specified using O'Sullivan and Mahan criteria [19, 20], seven studies used Carpenter-Coustan criteria [21-27]; three studies referenced the World Health Organization [28-30], one study referenced the Fourth International Gestational Diabetes Workshop criteria, and four studies did not specify the GDM diagnostic criteria used [31-34].

One study used amniotic fluid only [31], eight used maternal plasma only $[19,20,22,23,25,26,29,35]$, three used maternal serum only $[21,24,30]$, two used maternal urine only [28, 34], one used both amniotic fluid and urine [33], and one used both maternal urine and plasma [32]. Three studies examined metabolite profiles in the setting of other prenatal disorders $[28,33,34]$; however, only data on women with GDM without these potential confounders were abstracted from these studies. The analytical platforms used for metabolite detection included ion exchange chromatography [19], amino acid analyser [20], fluorescence polarisation immunoassay [21, 22], HPLC/dual wavelength spectrophotometry [23], Immunolite 2000 assay [24], ELISA-based assay [29, 30], HPLC/fluorescence spectroscopy [25], ultra-performance LC-MS [33], GC-MS [27] and ${ }^{1}$ H NMR spectroscopy [28, 31, $32,34]$. The methodologies, metabolites and regulation pattern ('upregulated', 'downregulated' or 'unchanged/not statistically significantly different') in each study are summarised in Table 1.

\section{Quality assessment}

Electronic supplementary material (ESM) Table 1 summarises the quality assessment process in accordance with the QUADOMICS tool [18]. Four of the 17 studies were classified as 'low quality' fulfilling fewer than 12 of the 16 criteria [18]. None of the studies stated whether the index test results were interpreted without knowledge of the results of the reference standard and the converse, thus failing criteria 12 and 13 of the QUADOMICS tool [18].

\section{Discussion}

\section{Specific metabolite groups}

Several investigations of metabolite profiles have identified specific biochemical groups to facilitate identification of potential mechanistic pathways for disease states. For example, a recent review has identified branched-chain and aromatic amino acids as highly significant predictors of the development of type 2 diabetes [12]. Metabolomic studies of pregnancies complicated by GDM have yielded inconsistent results, perhaps reflecting several potential contributory factors such as small participant populations, differing methodology of metabolomic analysis across studies, and variations in glycaemic control or treatment among participants. In this systematic review, the biomarkers most consistently associated with GDM are asymmetric dimethylarginine (ADMA) [25, 26, 29] and NEFAs [19, 27, 30, 35].

Branched-chain amino acids (BCAAs) BCAAs - valine, leucine and isoleucine - considered to be the most hydrophobic of the amino acids have been associated with risk factors for diabetes, including obesity [36] and insulin resistance [37]. In fact, studies have demonstrated elevated levels of BCAAs in non-diabetic individuals who later developed type 2 diabetes $[12,13]$. In contrast, although several studies have reported 
Fig. 1 Systematic review study selection algorithm. The 17 studies that fulfilled the inclusion/ exclusion criteria for this systematic review were selected from among 69 articles identified in the initial PubMed literature review. The process for selecting the 17 articles is displayed

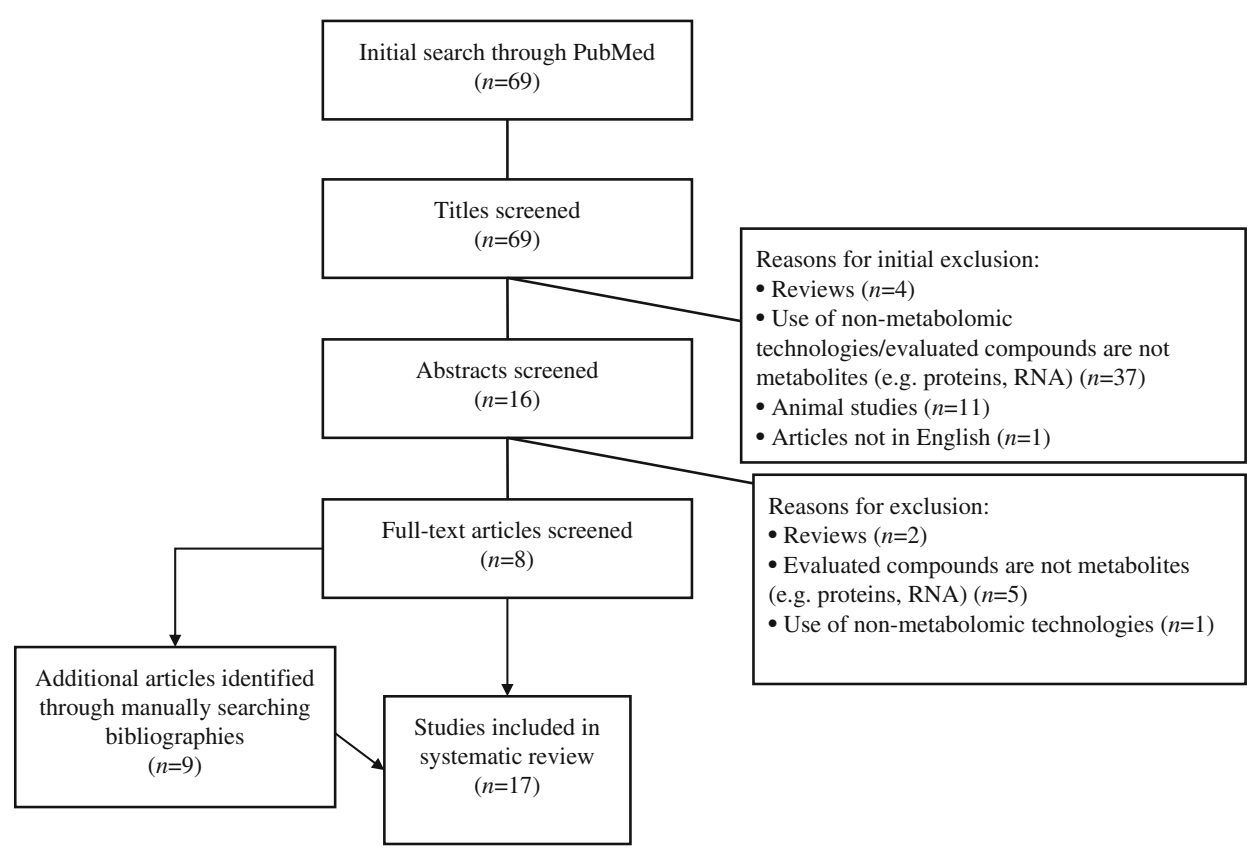

higher levels of BCAAs in women with GDM than controls $[19,20]$, these elevated levels have not been seen in all circumstances $[23,35]$. One of the earliest studies conducted on protein metabolism and GDM observed elevated levels of fasting and postprandial BCAAs in women with GDM compared with those without at 30-39 weeks' gestational age [19]. Similar results were reported for postprandial BCAAs but not fasting BCAAs at 32-36 weeks' gestational age [20]. More recent investigations by Cetin et al [23] and Pappa et al [35] with larger populations of women with GDM, however, reported no differences in circulating levels of BCAAs in maternal plasma for women with GDM compared with controls at 37-41 and 30-33 weeks' gestational age, respectively.

Aromatic amino acids The aromatic amino acids, tyrosine and phenylalanine, are characterised by the presence of an aromatic ring in their side chains. Along with BCAAs, aromatic amino acids have been implicated in the aetiology of liver failure [38]. Several large cohort studies on type 2 diabetes have also shown significantly increased levels of aromatic amino acids compared with controls [12, 39, 40]. With respect to GDM, higher levels of fasting and postprandial aromatic amino acids have been found by Butte et al [20] at 3236 weeks' gestational age in maternal plasma. However, Pappa et al observed no changes in aromatic amino acids at 30-33 weeks' gestational age, and Metzger et al [19] and Cetin et al [23] observed no changes in phenylalanine levels in maternal plasma at 30-39 or 37-41 weeks' gestational age, respectively.

Sulfur-containing compounds Sulfur-containing amino acids - methionine, cystine, cysteine and homocysteine- play a significant role in the maintenance of cellular systems. For example, defects in the regulation of sulfur concentrations in the body have been associated with vascular disease and cancer [41]. Butte et al [20] observed higher levels of cysteine in fasting and $2 \mathrm{~h}$ postprandial plasma levels for women with GDM compared with controls. Decreased methionine levels in maternal plasma have also been observed for women with GDM compared with controls [23]. Tarim et al [22], Seghieri et al [21] and Guven et al [24] found enhanced homocysteine levels in women with GDM at 24-28 weeks' gestational age. However, Idzior-Waluś et al [30] and Akturk et al [25] found no difference in homocysteine levels between women with GDM and controls at 26-32 and 32-39 weeks' gestational age, respectively. Further study in larger populations is needed to elucidate the effects of GDM on BCAAs, aromatic amino acids and sulfur-containing compounds.

ADMA ADMA, closely related to the amino acid, L-arginine, is a metabolic byproduct of repeated protein modification processes in the cytoplasm. It is considered a potential mediator of endothelial dysfunction [42], and clinical studies have observed increased levels of ADMA in individuals with several cardiometabolic diseases, including insulin resistance [43], hypercholesterolaemia [44], atherosclerosis [45], hypertension [46], chronic heart failure [47], type 2 diabetes mellitus [48], chronic renal failure [49] and histories of GDM [50-52]. Several clinical studies and reviews have also highlighted the potential role of ADMA as an independent predictor of the progression of atherosclerosis, cardiovascular death and all-cause mortality [42, 53-56].

Three studies of maternal plasma in women with GDM found increased levels of ADMA compared with women with 


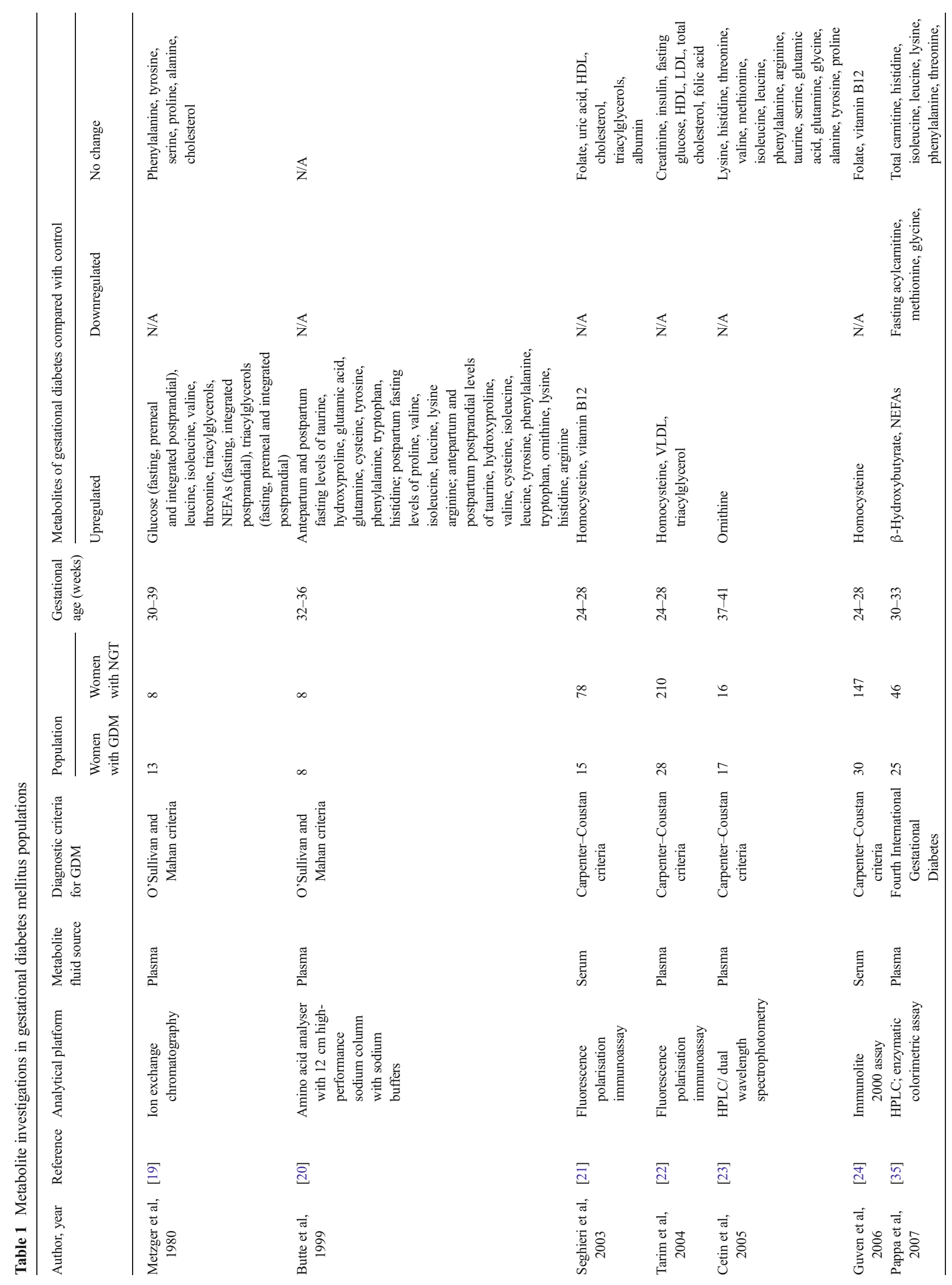




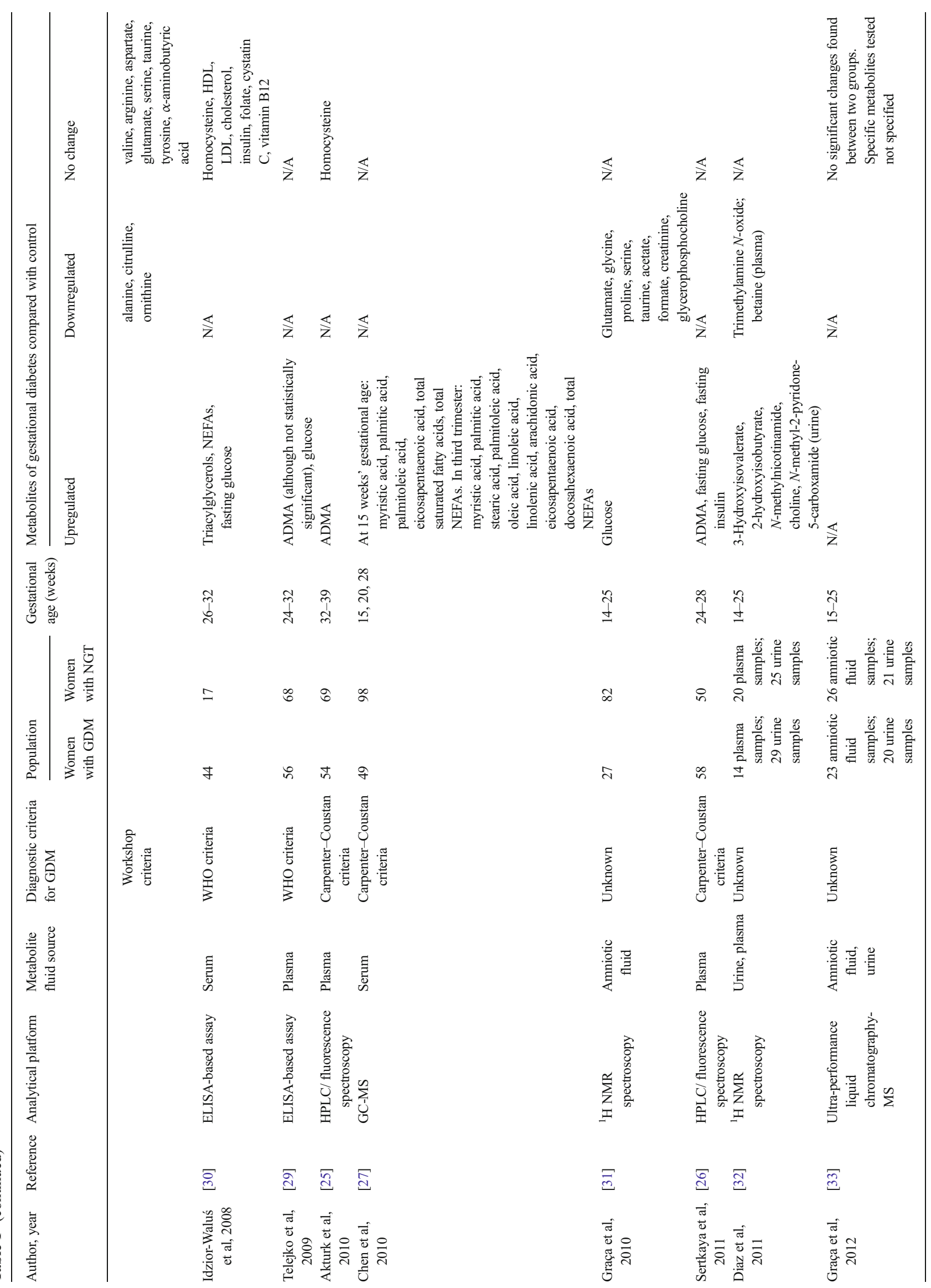




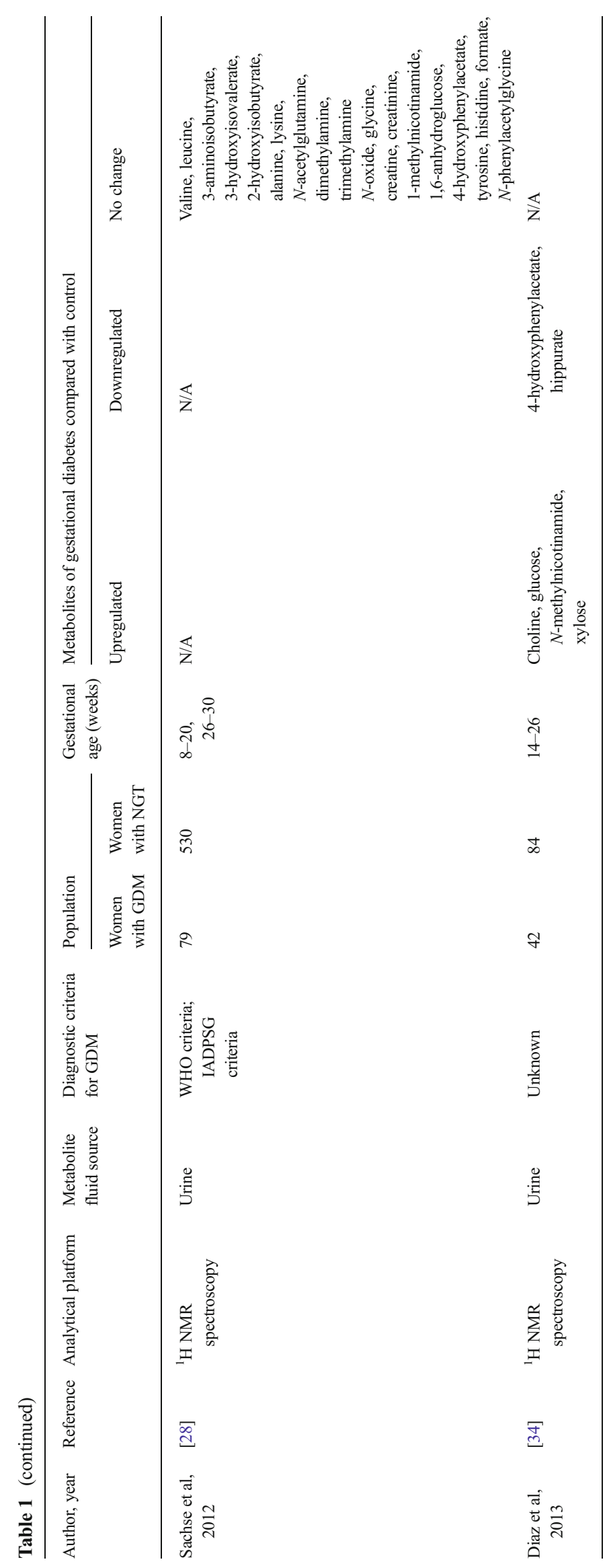


impaired glucose tolerance at gestational ages ranging from 24 to 39 weeks $[25,26,29]$. Sertkaya et al [26] observed that the increased ADMA correlated significantly with insulin sensitivity/resistance indices and moderately with $2 \mathrm{~h}$ insulin levels after an OGTT. The elevated levels of ADMA associated with GDM may be explained, in part, by the increased risk of concurrent hypertensive disorders during pregnancy $[57,58]$ and the increased subsequent cardiometabolic disease risk in women with GDM [3-5]. In addition, significant endothelial dysfunction has been observed among women with GDM compared with those with NGT during the third trimester of pregnancy [59], potentially implicating ADMA in the pathogenesis of this disease.

Other amino acids Additional amino acids have been examined in the context of GDM. Butte et al [20] also found higher fasting levels of taurine, hydroxyproline, glutamic acid and glutamine in maternal plasma in the third trimester among Hispanic women with insulin-treated GDM. These results suggest that, despite satisfactory maternal glycaemic control, protein metabolism reflected by elevated plasma amino acid concentrations is altered in women with GDM compared with those with NGT [20]. Another study by Cetin et al [23], which tested several amino acids in maternal plasma only, found higher levels of ornithine, an amino acid involved in the urea cycle, in women with GDM at delivery. However, to our knowledge, these results have not been replicated.

NEFAs NEFAs, the major components of triacylglycerols, are used as an energy source by many body tissues [60]. Adipose tissue lipolysis of triacylglycerols releases NEFAs and glycerol [60]. In addition, increased circulating levels of NEFAs have been well described in studies of insulin resistance and type 2 diabetes [61, 62]. Specifically, investigations on the inhibition of lipolysis by acipimox or nicotinic acid have shown improvement in meal tolerance and insulin sensitivity in patients with type 2 diabetes [63, 64].

Four studies have found upregulated levels of NEFAs in women with GDM in the third trimester of pregnancy $[19,27$, $30,35]$. Chen et al [27] observed that, at 15 weeks' gestational age, women with GDM had upregulated levels of essential and non-essential fatty acids, including myristic acid, palmitic acid, palmitoleic acid, eicosapentaenoic acid, total saturated fatty acids and total NEFAs, compared with those with NGT. During the third trimester, the same women with GDM had higher levels of myristic acid, palmitic acid, stearic acid, palmitoleic acid, oleic acid, linoleic acid, linolenic acid, eicosapentaenoic acid, docosahexaenoic acid, total saturated fatty acids, total monounsaturated fatty acids, polyunsatured fatty acids, and total NEFAs. Chen et al [27] also found that the dietary intake of polyunsaturated fatty acids was decreased, but saturated fatty acids were increased in women with GDM compared with control subjects. Because differences in fatty acid composition in women with GDM may exist because of diet or maternal adiposity levels, more research in this area is needed to elucidate potential mechanisms.

Other metabolites Biochemical pathways involving other metabolites have been implicated in the pathogenesis of type 2 diabetes, including triacylglycerols [65], acylcarnitines [66] and ketone bodies [67]. Specifically, non-fasting triacylglycerol levels have been associated with an increased risk of a number of cardiometabolic disorders, including myocardial infarction, ischaemic heart disease, hypertension and type 2 diabetes $[65,68]$. Increased acylcarnitine levels have been associated with insulin resistance $[69,70]$ and correlated with obesity and type 2 diabetes [66]. In addition, higher levels of circulating ketone bodies have been associated with obesity and type 2 diabetes and prediction of worsening hyperglycaemia [67].

Idzior-Waluś et al [30] and Tarim et al [22] observed higher levels of triacylglycerols in women with GDM, but these were not found to be significantly different by Seghieri et al [21]. Diaz et al [32] reported upregulated levels of 3hydroxyisovalerate, $\mathrm{N}$-methylnicotinamide and 2 hydroxyisobutyrate with lower levels of trimethylamine $\mathrm{N}$ oxide and betaine in the second trimester, but these results were not observed by Sachse et al [28] when examining maternal urine levels in the third trimester. Pappa et al [35] observed lower levels of fasting acylcarnitine esters and higher levels of fasting $\beta$-hydroxybutyrate in maternal plasma of women with GDM at 30-33 weeks' gestational age and suggested that there may be a shift from gluconeogenesis to ketone body production as the predominant energy source in GDM. However, these results have not been replicated by other investigative teams. Therefore, because there is still sufficient variability across observations, more research on these metabolites in larger population studies is warranted.

\section{Metabolomic studies in the prediction of GDM}

Five recent metabolomic studies of urine, amniotic fluid and/ or plasma have attempted to determine biomarkers for diagnosing GDM at 14-25 weeks' gestational age [28, 31-34], but findings have been inconsistent. Studies using NMR spectrometry have found increases in several metabolites in women with GDM, including acetate, creatine, creatinine, choline [34], 3-hydroxyisovalerate and hydroxyisobutyrate [32]. In addition, decreases in trimethylamine $\mathrm{N}$-oxide and betaine levels have also been observed in women with GDM [32]. Furthermore, in one study using NMR and LC-MS technologies, Graça et al [31] found small increases in several amino acids and organic acids. Yet, in a subsequent follow-up study, Graça et al [33] found no significant changes in metabolite profiles between women with GDM and controls. Sachse et al 
[28] also studied differences in metabolite levels between women with GDM and controls at 8-20 weeks' gestational age, but did not identify any reliable biomarkers for the diagnosis of GDM. Enhanced identification of maternal metabolites in the prediction of GDM necessitates further studies in larger, more racially/ethnically diverse populations.

\section{The future of metabolomics}

The application of metabolomics in diabetes research has provided the scientific community with new insights into the pathogenesis of diabetes. Several putative metabolic markers and associated pathways have been identified and validated in association with type 2 diabetes. Pathways implicated include those relevant to carbohydrate metabolism, lipid metabolism, amino acid metabolism (specifically with BCAAs and aromatic amino acids) and bile acids [71]. Our systematic review of women with GDM has revealed similar metabolic biomarkers and derangements in carbohydrate, lipid and amino acid metabolism. Women with GDM have consistently shown reduced peripheral insulin sensitivity [72], reduced suppression of hepatic glucose production [73], defects in pancreatic beta cell function [74], and an increase in the production of inflammatory cytokines [75], all of which have been shown to enhance chronic maternal insulin resistance [76]. In this systematic review, we suggest two additional pathways involving ADMA and NEFAs in the pathogenesis of GDM. Further research is needed to determine if the metabolite profiles associated with type 2 diabetes would also distinguish women with GDM from those with NGT. The elucidation of these relationships would be particularly informative because GDM is associated with an increased risk of subsequent type 2 diabetes [1]; GDM and type 2 diabetes potentially share a common genetic basis [77], and both disorders are characterised by insulin resistance and impaired insulin secretion [78].

Strengths of this systemic review include the rigorous and detailed way in which the literature was evaluated. However, the inconsistent elevation/reduction of some of the biomarkers and the small sample sizes of some of the study populations limit the ability to draw definitive conclusions from these data. Nonetheless, harnessing the benefits of metabolomics to gather information on GDM is a relatively new endeavour that requires further investigation in larger, more diverse populations. Therefore, it is important to examine current gaps in the literature as we carefully consider the best direction for subsequent research.

One conspicuous gap in the literature is the lack of large, well-defined cohorts that can offer robust analysis and comprehensive data on fasting and $2 \mathrm{~h}$ plasma samples. Using an OGTT in metabolomic studies of GDM will provide information on both anabolic and catabolic processes as well as indicate how effectively women with GDM process glucose loads during pregnancy. Recent investigations have shown that metabolomic analysis of samples from participants before and after an OGTT can be used to detect early shifts in metabolism during the progression from early insulin resistance to type 2 diabetes $[15,16]$. For example, Ho et al [15] studied a community-based population of 377 men and women with diabetes using LC-MS and found significant blunted changes after OGTT for $\beta$-hydroxybutyrate, isoleucine, lactate and pyridoxate in participants with insulin resistance compared with non-insulin-resistant individuals. Also, a pilot study of women with a history of GDM by Bentley-Lewis et al [17] reported metabolite decreases after an OGTT of similar magnitude to those found by Ho et al [15]. Moreover, Bentley-Lewis et al identified that the greatest changes in metabolite levels after an OGTT in women with a history of GDM was significantly associated with a longer duration of breastfeeding, higher BMI, increasing fasting glucose levels, race, adiponectin and parity, suggesting a relationship between metabolite profiles and behavioural and clinical characteristics [17]. Because the investigation of women with GDM often incorporates an OGTT to biochemically diagnose GDM at 24-28 weeks of gestation, future studies could include biological fluid collection for metabolomic analyses. In addition, consideration of clinical and behavioural factors of the population is critical to maximally characterise the impact of metabolite profile data [17].

Furthermore, the metabolomic methodology used should be optimised. Studies using a multiplatform approach, with both LC-MS and NMR spectroscopy for example, may provide a better understanding of metabolic derangements than studies only using one analytical tool. In addition, current studies of GDM have examined maternal urine, amniotic fluid, maternal plasma and maternal serum. Although the fluid used will depend in part on the outcome to be analysed, additional studies examining several biological fluids concurrently may provide a more complete overview of the metabolic and biochemical events that occur during a pregnancy complicated by GDM.

In addition, the timing of the investigation is critical. The majority of current metabolomic studies on GDM are conducted during the third trimester or after delivery. Yet, the ADA, ACOG, IADPSG and NIH recommend that women undergo OGTTs at the second trimester, and only women at high risk of type 2 diabetes are screened in the first trimester [1]. Consequently, there is a need to bridge the gap between clinical research and clinical practice, and to begin conducting metabolomic studies on specimens obtained during the earliest prenatal encounter. Metabolites identified in current studies of GDM require further research in larger, prospective, more racially/diverse populations before potential use in clinical practice. As we continue to consider the implications of emerging technologies in clinical practice, we advance towards more comprehensive models of care that can benefit the long-term health outcomes for mother and offspring. 
Acknowledgements The authors thank R. Walmer (Diabetes Research Center, Massachusetts General Hospital, Boston, MA, USA) for her assistance with manuscript preparation.

Funding This study was supported in part by the following grants awarded to RB-L: National Institutes of Health R03DK096152; the Robert Wood Johnson Foundation Harold Amos Medical Faculty Development Program; and the Massachusetts General Hospital Executive Committee on Research/Multicultural Affairs Office Physician-Scientist Development Award.

Duality of interest The authors declare that there is no duality of interest associated with this manuscript.

Contribution statement All authors reviewed the included studies, drafted and critically revised the manuscript for important intellectual content, and approved final version of the manuscript. JH and GX conducted the initial literature review and contributed to study design. RB-L conceived the study, is responsible for the integrity of the work as a whole, and is the guarantor of this work.

\section{References}

1. American Diabetes Association (2014) Standards of medical care in diabetes, 2014. Diabetes Care 37(Suppl 1):S14-S80

2. American Diabetes Association (2013) Standards of medical care in diabetes, 2013. Diabetes Care 36(Suppl 1):S11-S66

3. Shah BR, Retnakaran R, Booth GL (2008) Increased risk of cardiovascular disease in young women following gestational diabetes mellitus. Diabetes Care 31:1668-1669

4. Bentley-Lewis R (2009) Late cardiovascular consequences of gestational diabetes mellitus. Semin Reprod Med 27:322-329

5. Bentley-Lewis R, Powe C, Ankers E, Wenger J, Ecker J, Thadhani R (2014) Effect of race/ethnicity on hypertension risk subsequent to gestational diabetes mellitus. Am J Cardiol 113:1364-1370

6. Kelly BB, Narula J, Fuster V (2012) Recognizing global burden of cardiovascular disease and related chronic diseases. Mt Sinai J Med 79:632-640

7. Thadhani R, Powe CE, Tjoa ML et al (2010) First-trimester follistatin-like-3 levels in pregnancies complicated by subsequent gestational diabetes mellitus. Diabetes Care 33:664-669

8. Thadhani R, Wolf M, Hsu-Blatman K, Sandler L, Nathan D, Ecker JL (2003) First-trimester sex hormone binding globulin and subsequent gestational diabetes mellitus. Am J Obstet Gynecol 189:171-176

9. Smirnakis KV, Plati A, Wolf M, Thadhani R, Ecker JL (2007) Predicting gestational diabetes: choosing the optimal early serum marker. Am J Obstet Gynecol 196:410.e411-416, discussion 410.e416-417

10. Wolf M, Sandler L, Hsu K, Vossen-Smirnakis K, Ecker JL, Thadhani R (2003) First-trimester C-reactive protein and subsequent gestational diabetes. Diabetes Care 26:819-824

11. Savvidou M, Nelson SM, Makgoba M, Messow CM, Sattar N, Nicolaides K (2010) First-trimester prediction of gestational diabetes mellitus: examining the potential of combining maternal characteristics and laboratory measures. Diabetes 59: 3017-3022

12. Friedrich N (2012) Metabolomics in diabetes research. J Endocrinol 215:29-42

13. Roberts LD, Koulman A, Griffin JL (2014) Towards metabolic biomarkers of insulin resistance and type 2 diabetes: progress from the metabolome. Lancet Diabetes Endocrinol 2:65-75
14. Fanos V, Atzori L, Makarenko K, Melis GB, Ferrazzi E (2013) Metabolomics application in maternal-fetal medicine. Biomed Res Int 2013:720514

15. Ho JE, Larson MG, Vasan RS et al (2013) Metabolite profiles during oral glucose challenge. Diabetes 62:2689-2698

16. Shaham O, Wei R, Wang TJ et al (2008) Metabolic profiling of the human response to a glucose challenge reveals distinct axes of insulin sensitivity. Mol Syst Biol 4:214

17. Bentley-Lewis R, Xiong G, Lee H, Yang A, Huynh J, Kim C (2014) Metabolomic analysis reveals amino acid responses to an oral glucose tolerance test in women with prior history of gestational diabetes mellitus. J Clin Transl Endocrinol 1:38-43

18. Lumbreras B, Porta M, Márquez S, Pollán M, Parker LA, HernándezAguado I (2008) QUADOMICS: an adaptation of the Quality Assessment of Diagnostic Accuracy Assessment (QUADAS) for the evaluation of the methodological quality of studies on the diagnostic accuracy of '-omics'-based technologies. Clin Biochem 41: $1316-1325$

19. Metzger BE, Phelps RL, Freinkel N, Navickas IA (1980) Effects of gestational diabetes on diurnal profiles of plasma glucose, lipids, and individual amino acids. Diabetes Care 3:402-409

20. Butte NF, Hsu HW, Thotathuchery M, Wong WW, Khoury J, Reeds P (1999) Protein metabolism in insulin-treated gestational diabetes. Diabetes Care 22:806-811

21. Seghieri G, Breschi MC, Anichini R et al (2003) Serum homocysteine levels are increased in women with gestational diabetes mellitus. Metabolism 52:720-723

22. Tarim E, Bagis T, Kilicdag E et al (2004) Elevated plasma homocysteine levels in gestational diabetes mellitus. Acta Obstet Gynecol Scand 83:543-547

23. Cetin I, de Santis MS, Taricco E et al (2005) Maternal and fetal amino acid concentrations in normal pregnancies and in pregnancies with gestational diabetes mellitus. Am J Obstet Gynecol 192:610-617

24. Guven MA, Kilinc M, Batukan C, Ekerbicer HC, Aksu T (2006) Elevated second trimester serum homocysteine levels in women with gestational diabetes mellitus. Arch Gynecol Obstet 274:333-337

25. Akturk M, Altinova A, Mert I et al (2010) Asymmetric dimethylarginine concentrations are elevated in women with gestational diabetes. Endocrine 38:134-141

26. Sertkaya AC, Kafkasli A, Turkcuoglu I, Karabulut AB (2011) Asymmetric dimethylarginine level in hyperglycemic gestation. Endocrine 40:237-242

27. Chen X, Scholl TO, Leskiw M, Savaille J, Stein TP (2010) Differences in maternal circulating fatty acid composition and dietary fat intake in women with gestational diabetes mellitus or mild gestational hyperglycemia. Diabetes Care 33:2049-2054

28. Sachse D, Sletner L, Mørkrid K et al (2012) Metabolic changes in urine during and after pregnancy in a large, multiethnic population-based cohort study of gestational diabetes. PLoS One 7:e52399

29. Telejko B, Zonenberg A, Kuzmicki M et al (2009) Circulating asymmetric dimethylarginine, endothelin-1 and cell adhesion molecules in women with gestational diabetes. Acta Diabetol 46:303-308

30. Idzior-Waluś B, Cyganek K, Sztefko K et al (2008) Total plasma homocysteine correlates in women with gestational diabetes. Arch Gynecol Obstet 278:309-313

31. Graça G, Duarte IF, Barros AS et al (2010) Impact of prenatal disorders on the metabolic profile of second trimester amniotic fluid: a nuclear magnetic resonance metabonomic study. J Proteome Res 9: 6016-6024

32. Diaz SO, Pinto J, Graça G et al (2011) Metabolic biomarkers of prenatal disorders: an exploratory NMR metabonomics study of second trimester maternal urine and blood plasma. J Proteome Res 10:3732-3742

33. Graça G, Goodfellow BJ, Barros AS et al (2012) UPLC-MS metabolic profiling of second trimester amniotic fluid and maternal urine 
and comparison with NMR spectral profiling for the identification of pregnancy disorder biomarkers. Mol Biosyst 8:1243-1254

34. Diaz SO, Barros AS, Goodfellow BJ et al (2013) Second trimester maternal urine for the diagnosis of trisomy 21 and prediction of poor pregnancy outcomes. J Proteome Res 12:2946-2957

35. Pappa KI, Vlachos G, Theodora M, Roubelaki M, Angelidou K, Antsaklis A (2007) Intermediate metabolism in association with the amino acid profile during the third trimester of normal pregnancy and diet-controlled gestational diabetes. Am J Obstet Gynecol 196: 65.e61-65

36. McCormack SE, Shaham O, McCarthy MA et al (2013) Circulating branched-chain amino acid concentrations are associated with obesity and future insulin resistance in children and adolescents. Pediatr Obes 8:52-61

37. Newgard CB, An J, Bain JR et al (2009) A branched-chain amino acid-related metabolic signature that differentiates obese and lean humans and contributes to insulin resistance. Cell Metab 9:311-326

38. Dejong CH, van de Poll MC, Soeters PB, Jalan R, Olde Damink SW (2007) Aromatic amino acid metabolism during liver failure. J Nutr 137:1579S-1585S, discussion 1597S-1598S

39. Cheng S, Rhee EP, Larson MG et al (2012) Metabolite profiling identifies pathways associated with metabolic risk in humans. Circulation 125:2222-2231

40. Wang TJ, Larson MG, Vasan RS et al (2011) Metabolite profiles and the risk of developing diabetes. Nat Med 17:448-453

41. Townsend DM, Tew KD, Tapiero H (2004) Sulfur containing amino acids and human disease. Biomed Pharmacother 58:47-55

42. Sibal L, Agarwal SC, Home PD, Boger RH (2010) The role of asymmetric dimethylarginine (ADMA) in endothelial dysfunction and cardiovascular disease. Curr Cardiol Rev 6:82-90

43. Toutouzas K, Riga M, Stefanadi E, Stefanadis C (2008) Asymmetric dimethylarginine (ADMA) and other endogenous nitric oxide synthase (NOS) inhibitors as an important cause of vascular insulin resistance. Horm Metab Res 40:655-659

44. Böger RH, Bode-Böger SM, Szuba A et al (1998) Asymmetric dimethylarginine (ADMA): a novel risk factor for endothelial dysfunction: its role in hypercholesterolemia. Circulation 98:1842-1847

45. Miyazaki H, Matsuoka H, Cooke JP et al (1999) Endogenous nitric oxide synthase inhibitor: a novel marker of atherosclerosis. Circulation 99:1141-1146

46. Surdacki A, Nowicki M, Sandmann J et al (1999) Reduced urinary excretion of nitric oxide metabolites and increased plasma levels of asymmetric dimethylarginine in men with essential hypertension. J Cardiovasc Pharmacol 33:652-658

47. Usui M, Matsuoka H, Miyazaki H, Ueda S, Okuda S, Imaizumi T (1998) Increased endogenous nitric oxide synthase inhibitor in patients with congestive heart failure. Life Sci 62:2425-2430

48. Abbasi F, Asagmi T, Cooke JP et al (2001) Plasma concentrations of asymmetric dimethylarginine are increased in patients with type 2 diabetes mellitus. Am J Cardiol 88:1201-1203

49. Böger RH, Zoccali C (2003) ADMA: a novel risk factor that explains excess cardiovascular event rate in patients with end-stage renal disease. Atheroscler Suppl 4:23-28

50. Gumus II, Kargili A, Kaygusuz I et al (2012) The association between serum asymmetric dimethyl arginine levels and a history of gestational diabetes among healthy women. Blood Coagul Fibrinolysis 23:391395

51. Pleiner J, Mittermayer F, Langenberger H et al (2007) Impaired vascular nitric oxide bioactivity in women with previous gestational diabetes. Wien Klin Wochenschr 119:483-489

52. Mittermayer F, Mayer BX, Meyer A et al (2002) Circulating concentrations of asymmetrical dimethyl-L-arginine are increased in women with previous gestational diabetes. Diabetologia 45:1372-1378

53. Lu TM, Ding YA, Lin SJ, Lee WS, Tai HC (2003) Plasma levels of asymmetrical dimethylarginine and adverse cardiovascular events after percutaneous coronary intervention. Eur Heart J 24:1912-1919
54. Valkonen VP, Päivä H, Salonen JT et al (2001) Risk of acute coronary events and serum concentration of asymmetrical dimethylarginine. Lancet 358:2127-2128

55. Meinitzer A, Seelhorst U, Wellnitz B et al (2007) Asymmetrical dimethylarginine independently predicts total and cardiovascular mortality in individuals with angiographic coronary artery disease (the Ludwigshafen Risk and Cardiovascular Health study). Clin Chem 53:273-283

56. Böger RH (2005) Asymmetric dimethylarginine (ADMA) and cardiovascular disease: insights from prospective clinical trials. Vasc Med 10(Suppl 1):S19-S25

57. Bryson CL, Ioannou GN, Rulyak SJ, Critchlow C (2003) Association between gestational diabetes and pregnancy-induced hypertension. Am J Epidemiol 158:1148-1153

58. Barden A, Singh R, Walters BN, Ritchie J, Roberman B, Beilin LJ (2004) Factors predisposing to pre-eclampsia in women with gestational diabetes. J Hypertens 22:2371-2378

59. Paradisi G, Biaggi A, Ferrazzani S, de Carolis S, Caruso A (2002) Abnormal carbohydrate metabolism during pregnancy: association with endothelial dysfunction. Diabetes Care 25:560-564

60. Wolfe RR (1998) Fat metabolism in exercise. Adv Exp Med Biol 441:147-156

61. Boden G, Shulman GI (2002) Free fatty acids in obesity and type 2 diabetes: defining their role in the development of insulin resistance and beta-cell dysfunction. Eur J Clin Invest 32(Suppl 3):14-23

62. Boden G (2003) Effects of free fatty acids (FFA) on glucose metabolism: significance for insulin resistance and type 2 diabetes. Exp Clin Endocrinol Diabetes 111:121-124

63. Fulcher GR, Catalano C, Walker M et al (1992) A double blind study of the effect of acipimox on serum lipids, blood glucose control and insulin action in non-obese patients with type 2 diabetes mellitus. Diabet Med 9:908-914

64. Vaag A, Skött P, Damsbo P, Gall MA, Richter EA, Beck-Nielsen H (1991) Effect of the antilipolytic nicotinic acid analogue acipimox on whole-body and skeletal muscle glucose metabolism in patients with non-insulin-dependent diabetes mellitus. J Clin Invest 88:1282-1290

65. Bitzur R, Cohen H, Kamari Y, Shaish A, Harats D (2009) Triglycerides and HDL cholesterol: stars or second leads in diabetes? Diabetes Care 32(Suppl 2):S373-S377

66. Mihalik SJ, Goodpaster BH, Kelley DE et al (2010) Increased levels of plasma acylcarnitines in obesity and type 2 diabetes and identification of a marker of glucolipotoxicity. Obesity (Silver Spring) 18: 1695-1700

67. Mahendran Y, Vangipurapu J, Cederberg H et al (2013) Association of ketone body levels with hyperglycemia and type 2 diabetes in 9 , 398 Finnish men. Diabetes 62:3618-3626

68. Nordestgaard BG, Benn M, Schnohr P, Tybjaerg-Hansen A (2007) Nonfasting triglycerides and risk of myocardial infarction, ischemic heart disease, and death in men and women. JAMA 298:299-308

69. Hoppel CL, Genuth SM (1980) Carnitine metabolism in normalweight and obese human subjects during fasting. Am J Physiol 238:E409-E415

70. Soeters MR, Sauerwein HP, Duran M et al (2009) Muscle acylcarnitines during short-term fasting in lean healthy men. Clin Sci (Lond) 116:585-592

71. Lu J, Xie G, Jia W (2013) Metabolomics in human type 2 diabetes research. Front Med 7:4-13

72. Catalano PM (2014) Trying to understand gestational diabetes. Diabet Med 31:273-281

73. Catalano PM, Huston L, Amini SB, Kalhan SC (1999) Longitudinal changes in glucose metabolism during pregnancy in obese women with normal glucose tolerance and gestational diabetes mellitus. Am J Obstet Gynecol 180:903-916 
74. Buchanan TA (2001) Pancreatic B cell defects in gestational diabetes: implications for the pathogenesis and prevention of type 2 diabetes. J Clin Endocrinol Metab 86:989-993

75. Desoye G, Hauguel-de Mouzon S (2007) The human placenta in gestational diabetes mellitus. The insulin and cytokine network. Diabetes Care 30(Suppl 2):S120-S126

76. Barbour LA, McCurdy CE, Hernandez TL, Kirwan JP, Catalano PM, Friedman JE (2007) Cellular mechanisms for insulin resistance in normal pregnancy and gestational diabetes. Diabetes Care 30(Suppl 2):S112-S119

77. Robitaille J, Grant AM (2008) The genetics of gestational diabetes mellitus: evidence for relationship with type 2 diabetes mellitus. Genet Med 10:240-250

78. Ben-Haroush A, Yogev Y, Hod M (2004) Epidemiology of gestational diabetes mellitus and its association with type 2 diabetes. Diabet Med 21:103-113 